\title{
THE ESSENTIAL BOUNDARY OF CERTAIN SETS
}

\author{
A. M. BRUCKNER ${ }^{1}$, ROY O. DAVIES AND C. GOFFMAN ${ }^{1}$
}

\begin{abstract}
The essential boundary of a measurable set is related to the de Giorgi perimeter and was introduced by Vol'pert in his "improvement" of Federer's work.

For a totally disconnected compact set of positive measure in $n$ space the essential boundary can be of Hausdorff $n-1$ dimension but cannot have $\sigma$ finite $(n-1)$ measure.
\end{abstract}

Let $E \subset R^{n}$ be a measurable set with respect to Lebesgue measure. It is said to be of finite perimeter if all the partial derivatives $\mu_{1}(A), \ldots, \mu_{n}(A)$ of its characteristic function $\chi$ are totally finite measures over the Borel sets $A \subset R^{n}$. It is well known that $\mu_{i}=\mu_{i}\left(R^{n}\right)=\int v_{i}$, where $v_{i}$ is the infinum of the variations in $x_{i}$ of all functions equivalent to $\chi$ and the integration is over the $(n-1)$-space orthogonal to the $x_{i}$ axis, $O x_{i}$. The value of the perimeter is then the variation measure of the vector valued measure $\left(\mu_{1}(A), \ldots, \mu_{n}(A)\right)$, evaluated for $R^{n}$.

It was shown by Federer [1] that the perimeter is equal to the $(n-1)$-measure of a set that he called the reduced boundary of $E$, consisting of those points at which a certain generalized normal exists. Specifically, a point $p$ is in the reduced boundary of $E$ if there is an $(n-1)$-plane $\pi$ through $p$ such that the part of $E$ on one side of $\pi$ has density 0 at $p$, and the part of $C E$ on the other side of $\pi$ has density 0 at $p$. (The $k$-measure $\lambda_{k}(E)$ of a set in $R^{n}$ will mean the $k$-dimensional Hausdorff measure, normalized so that the $k$-dimensional unit cube has measure 1.) Vol'pert [2] showed that in the result the reduced boundary may be replaced by the essential boundary $\partial_{e}(E)$, consisting of those points of $R^{n}$ which are neither points of density 1 nor points of density 0 of $E$. Clearly, the essential boundary of $E$ contains the restricted boundary. Vol'pert's remarkable theorem asserts that, if $E$ has finite perimeter, then the Hausdorff $(n-1)$-measure of the restricted and essential boundaries are equal. The Lebesgue theorem guarantees that $\partial_{e}(E)$ is of $n$-measure zero, but in its perimeter role $\partial_{e}(E)$ has more of an $(n-1)$-dimensional flavor, and evidently coincides with the ordinary boundary $\partial(E)$ when this is a sufficiently smooth surface.

Going to the opposite extreme, in this note we shall discuss the possible nature of $\partial_{e}(E)$, with respect to $(n-1)$-measure, when $E$ is a nowhere dense set of positive measure in $R^{n}$.

Received by the editors October 23, 1984.

1980 Mathematics Subject Classification. Primary 28A75.

${ }^{1}$ These authors were supported in part by grants from the National Science Foundation. Part of the work was accomplished while the third author was visiting the University of California at Santa Barbara during a Special Year in Real Analysis. 
In the case $n=1$, it is easy to see that $\partial_{e}(E)$ is then of non- $\sigma$-finite 0 -measure; in other words, it is an uncountable set. In fact, since there exists an interval in which the relative measure of $E$ is greater than $\frac{1}{2}$, while intervals disjoint from $E$ are dense in $R$, by continuity we can find two disjoint compact intervals $I(0), I(1)$ such that

$$
0<\lambda_{1}(E \cap I(0))=\lambda_{1}(E \cap I(1))=\frac{1}{2} \lambda_{1}(I(0))=\frac{1}{2} \lambda_{1}(I(1))<\frac{1}{4} .
$$

Similarly, inside each $I(i), i=0,1$, we can find two disjoint compact intervals $I(i, 0), I(i, 1)$ of length less than $\frac{1}{4}$, in each of which the relative measure of $E$ is again $\frac{1}{2}$, and so on. Now $\partial_{e}(E)$ evidently contains the uncountable set

$$
\bigcap_{r=1}^{\infty} \bigcup\left\{I\left(\varepsilon_{1}, \ldots, \varepsilon_{r}\right): \varepsilon_{\rho}=0 \text { or } 1\right\} .
$$

In $R^{n}, n \geqslant 2$, a nowhere dense closed set may be of positive $n$-measure and yet have finite perimeter. Indeed, let $\sigma_{0}$ denote the open unit ball, and let $\sigma_{1}, \sigma_{2}, \ldots$, be a sequence of disjoint open balls in $\sigma_{0}$ such that $U_{m \geqslant 1} \sigma_{m}$ is dense in $\sigma_{0}$, but of smaller $n$-measure than $\sigma_{0}$, and $\sum_{m \geqslant 1} c_{m}<\infty$, where $c_{m}$ is the circumference of $\sigma_{m}$. Then $F=\bar{\sigma}_{0} \backslash \bigcup_{m \geqslant 1} \sigma_{m}$ is a nowhere dense closed set of positive $n$-measure; however, it is clear that for the characteristic function of $F$ we have $\mu_{i} \leqslant \sum_{m} \geqslant 0 c_{m}$ for $i=1, \ldots, n$, so that $F$ has finite perimeter. It can of course be established directly that the $(n-1)$-measure of $\partial_{e}(F)$ is finite but this is appreciably more difficult.

As can be seen from Theorem 3 below, it is possible for a set such as $F$ to have finite perimeter only because (although nowhere dense) it is far from being totally disconnected; indeed, in a certain sense, for most straight lines that intersect $F$ in positive 1-measure, the intersection must consist largely of complete intervals. In fact, we shall show that if $F$ is any closed set, then on almost every straight line in any given direction the essential boundary $\partial_{e}(F)$ includes an important part of the topological boundary of $F$, relative to the line. Consequently, when $F$ is totally disconnected the set $\partial_{e}(F)$ must be of non- $\sigma$-finite $(n-1)$-measure.

We first point out that in this case, $\partial_{e}(F)$ is not necessarily of Hausdorff dimension greater than $n-1$.

THEOREM 1. In $R^{n}$ there exists a totally disconnected compact set $F$ of positive $n$-measure for which $\partial_{e}(F)$ is of Hausdorff dimension $n-1$.

Proof. Let $C$ denote the $n$-dimensional unit cube $[0,1]^{n}$. Let $\theta_{1}, \theta_{2}, \ldots$ be a sequence of positive numbers such that the series $\sum_{r}\left(\left(2 r^{2}+1\right) \theta_{r}\right)^{\alpha}$ converges for every $\alpha>0$ and $\sum_{r} \theta_{r}<1 / n$; for example, let $\theta_{r}=1 / n\left(2 r^{2}+1\right) 2^{r}$.

Let $q_{1}, q_{2}, \ldots$ be an enumeration of all rational numbers in [0,1]. Let $I_{r}$ denote the open interval $\left(a_{r}, b_{r}\right)$ with center at $q_{r}$ and of length $\theta_{r}$. Let $S_{r 1}$ denote the slab $I_{r} \times[0,1] \times \cdots \times[0,1]$, and define $S_{r i}$ similarly for $i=2, \ldots, n$ as the product of the interval $I_{r}$ placed on the axis $O x_{i}$ with a unit cube in the (n-1)-space orthogonal to $O_{x i}$; let $S_{r}=S_{r 1} \cup \cdots \cup S_{r n}$ and $F=C \backslash \cup_{r} S_{r}$. Then $F$ is a totally disconnected compact set with

$$
\lambda_{n}(F) \geqslant \lambda_{n}(C)-\sum_{r} \lambda_{n}\left(S_{r} \cap C\right) \geqslant 1-n \sum_{r} \theta_{r}>0 .
$$


Now let $T_{r i}$ denote the slab consisting of all points at a distance less than $r^{2} \theta_{r}$ from $S_{r i}$, put $T_{r}=T_{r 1} \cup \cdots \cup T_{r n}$, and let $H=\lim \sup \left(T_{r} \cap C\right)$. Then $H$ is of Hausdorff dimension $n-1$, because for every $R$ we have $H \subset \bigcup_{r \geqslant R}\left(T_{r} \cap C\right)$, for each $r, i$ the set $T_{r i} \cap C$ can be covered by $O\left(\left[1 /\left(2 r^{2}+1\right) \theta_{r}\right]^{r-1}\right)$ cubes of side $\left(2 r^{2}+1\right) \theta_{r}$, and for every $\beta>n-1$,

$$
\sum_{r \geqslant R}\left(\frac{1}{\left(2 r^{2}+1\right) \theta_{r}}\right)^{n}\left(\left(2 r^{2}+1\right) \theta_{r}\right)^{\beta} \rightarrow 0 \quad \text { as } R \rightarrow \infty .
$$

It will follow from Theorem 3 that $\partial_{e}(F)$ has non- $\sigma$-finite $(n-1)$-measure, so it is now enough to show that $\partial_{e}(F) \subset H$, and hence enough to show that if $x \in F \backslash H$ then the density of $\bigcup_{r} S_{r}$ at $x$ is zero. Let $x=\left(x_{1}, \ldots, x_{n}\right) \in F \backslash H$. It is enough to show, for example, that the density of $\bigcup_{r} S_{r}$ at $x$ in the orthant $\left\{y: y_{i}>x_{i}\right.$ for $i=1, \ldots, n\}$ is zero.

Given $\varepsilon>0$, choose $R$ so large that $\sum_{r \geqslant R} 1 / r^{2}<\varepsilon / n$. Since $x \notin H$, we can choose $Q$ so that $x \notin \cup_{r \geqslant Q} T_{r}$, and we may suppose $Q \geqslant R$. Therefore,

$$
\sum_{r \geqslant Q} \frac{1}{r^{2}}<\frac{\varepsilon}{n}
$$

We can choose $\delta>0$ so small that $\bigcup_{r=1}^{Q-1} S_{r}$ does not meet the cube

$$
C_{\delta}=\left\{y: 0 \leqslant y_{i}<x_{i}<\delta \text { for } i=1, \ldots, n\right\} .
$$

Given $0<h<\delta$, we shall show (establishing the conclusion) that

$$
\lambda_{n}\left[C_{h} \cap \bigcup_{r} S_{r}\right] \leqslant \varepsilon \lambda_{n}\left(C_{h}\right) .
$$

Consider any slab $S_{r i}$ such that $S_{r i} \cap C_{h} \neq \varnothing$; then $r \geqslant Q$. Without loss of generality we may suppose that $i=1$. Because $x \notin T_{r 1}$, but $S_{r 1} \cap C \neq \varnothing$, we have $x_{1}<a_{r}-r^{2} \theta_{r}<a_{r}<x_{1}+h$, and therefore

$$
\lambda_{1}\left[\left(x_{1}, x_{1}+h\right) \cap I_{r}\right] \leqslant \lambda_{1}\left(I_{r}\right)=\theta_{r}=\left(1 / r^{2}\right)\left[a_{r}-\left(a_{r}-r^{2} \theta_{r}\right)\right]<\left(1 / r^{2}\right) h,
$$

from which it follows that $\lambda_{n}\left(C_{h} \cap S_{r 1}\right) \leqslant\left(1 / r^{2}\right) \lambda_{n}\left(C_{h}\right)$. By (1) this implies the required result (2).

We proceed to show that for a totally disconnected compact set $K$ of positive $n$-measure the set $\partial_{e}(K)$ is of non- $\sigma$-finite $(n-1)$-measure. We first obtain a result on arbitrary measurable sets. Let $E \subset R^{n}$ be measurable with $\lambda_{n}(E)>0$. We adjust $E$ in the $x_{1}$ direction as follows: on every open interval in any line parallel to $O x_{1}$, if $E$ has zero linear measure in the interval, transfer the entire interval to $C E$, and if $C E$ has linear measure zero in the interval, place the entire interval in $E$. By the linear density theorem, this adjustment only changes $E$ by a set of $n$-measure zero. Such an adjusted set may be called $x_{1}$-smooth. Thus, if $E$ is $x_{1}$-smooth, then for every open interval $I$ situated in a line parallel to $O x_{1}$ we have that $\lambda_{1}(E \cap I)=0$ implies $E \cap I=\varnothing$ and $\lambda_{1}((C E) \cap I)=0$ implies $(C E) \cap I=\varnothing$.

In what follows it will be convenient to regard a typical point in $R^{n}$ as $(x ; y)$ where $x \in R$ and $y \in R^{n-1}$. For any set $E \subset R^{n}$ and any $y \in R^{n-1}$, by $\partial\left(E^{y}\right)$ we shall mean the ordinary boundary in $R$ of the section $E^{y}=\{x:(x ; y) \in E\}$, that 
is, $\partial\left(E^{y}\right)=\overline{E^{v}} \cap \overline{\left(R \backslash E^{y}\right)}$. By $\underline{d}(E, p)$ and $\bar{d}(E, p)$, where $E \subset R^{n}$ and $p \in R^{n}$, we mean the respective limits as $\delta \rightarrow 0+$ of the infinum and supremum of $\left\{\lambda_{n}(E \cap S) / \lambda_{n}(S): S \in \mathscr{S}(\delta, p)\right\}$ where $\mathscr{S}(\delta, p)$ is the collection of all open cubes with sides parallel to the axes containing $p$ and with side of length less than $\delta$. A subset $A$ of a set $B$ is said to be residual in $B$ if $B \backslash A$ is the union of countably many sets each of which is nowhere dense in $B$.

THEOREM 2. If $E$ is any $x_{1}$-smooth measurable set in $R^{n}$, then for almost all $y \in R^{n-1}$ the set

$$
\{x: \underline{d}(E,(x ; y))=0 \text { and } \bar{d}(E,(x ; y))=1\} \cap\left(E^{y}\right)
$$

is residual in $\partial\left(E^{y}\right)$.

Proof. It is sufficient to prove that for almost all $y \in R^{n-1}$ the set $\{x$ : $\underline{d}(E,(x ; y))=0\} \cap \partial\left(E^{y}\right)$ is residual in $\partial\left(E^{y}\right)$, because this same result applied to $C E$ will show that $\{x: \underline{d}(C E,(x ; y))=0\} \cap \partial(C E)^{y}$ is also residual in $\partial\left((C E)^{v}\right)=$ $\partial\left(E^{y}\right)$ for almost all $y \in R^{n-1}$.

Given any positive integer $r$, let $A(r)$ denote the union of all open cubes $C$ such that $\lambda_{n}(C)<r^{-n}$ and $\lambda_{n}(E \cap C)<r^{-1} \lambda_{n}(C)$. It will be enough to show that for almost all $y \in R^{n-1}$ the set $A(r)^{y} \cap \partial\left(E^{y}\right)$ is dense in $\partial\left(E^{y}\right)$, since $A(r)$ is open and therefore $\partial\left(E^{y}\right) \backslash A(r)^{y}$ will then be nowhere dense for each $r$, and consequently $\{x: \underline{d}(E,(x ; y))>0\} \cap \partial\left(E^{y}\right)$ is of the first category in $\partial\left(E^{y}\right)$.

We henceforth regard $r$ as fixed and write $A$ for $A(r)$. Suppose, if possible, that for a set of $y \in R^{n-1}$ of positive outer $(n-1)$-measure the set $A^{y} \cap \partial\left(E^{y}\right)$ is not dense in $\partial\left(E^{y}\right)$; we shall derive a contradiction. For each such $y$ there exists a nonempty open interval $I \subset R$ with rational endpoints such that

$$
I \cap \partial\left(E^{y}\right) \neq \varnothing \quad \text { and } I \cap \partial\left(E^{y}\right) \cap A^{y}=\varnothing .
$$

Hence there exists a set $Z \subset R^{n-1}$ of positive outer $(n-1)$-measure and a fixed nonempty open interval $I \subset R$ such that (3) holds for all $y \in Z$. Whenever (3) holds, we have $I \cap(C E)^{y} \neq \varnothing$ and therefore $\lambda_{1}^{*}\left[I \cap(C E)^{y}\right]>0$, because $E$ is $x_{1}$-smooth. Since almost all points of $C E$ are points of density 1 for $C E$, it follows that for almost every $z \in Z$ there exists $x \in I$ for which $d(E,(x ; z))=0$. Choose any such point $z \in Z$ which is also a point of outer $(n-1)$-density 1 for $Z$, choose $h>0$ so small that $\lambda_{n-1}^{*}(Z \cap K)>\left(1-\frac{1}{2} r^{-1}\right) \lambda_{n-1}(K)$ for every open cube $K \subset$ $R^{n-1}$ of side less than $h$, containing $z$, and let $x_{0} \in I$ be such that $d\left(E,\left(x_{0} ; z\right)\right)=0$.

Let $C_{0}$ be an open cube of side less than $\min \left(h, r^{-1}\right)$ with $\left(x_{0} ; z\right) \in C_{0}$, and

$$
\lambda_{n}\left(E \cap C_{0}\right)<\frac{1}{2} r^{-1} \lambda_{n}\left(C_{0}\right),
$$

and $C_{0}=J_{0} \times K$ where $J_{0} \subset I$ and $K \subset R^{n-1}$; thus $z \in K$. Since $z \in Z$, the set $I \cap \partial\left(E^{y}\right)$ is nonempty; let $x^{\prime}$ be any point of it. Translate $J_{0}$ a distance $t$ (to the left or right) to a position $J_{t} \subset I$ for which $x^{\prime} \in J_{t}$. Since $\left(x^{\prime} ; z\right) \notin A$, the cube $C_{t}=J_{t} \times K$ satisfies $\lambda_{n}\left(E \cap C_{t}\right) \geqslant r^{-1} \lambda_{n}\left(C_{t}\right)$. In view of (4) there exists a least value of $t>0$ for which the last inequality holds; denoting it by $\eta$, we have

$$
\lambda_{n}\left(E \cap C_{\eta}\right)=r^{-1} \lambda_{n}\left(C_{\eta}\right)
$$


and

$$
\lambda_{n}\left(E \cap C_{\tau}\right)<r^{-1} \lambda_{n}\left(C_{\tau}\right) \text { for } 0<\tau<\eta .
$$

By (4) and Fubini's theorem, $\lambda_{n-1}(W)<\frac{1}{2} r^{-1} \lambda_{n-1}(K)$, where $W=\{y \in K$ : $\left.\left(C_{0} \cap C E\right)^{y}=\varnothing\right\}$. Now consider any $y \in K \backslash W$ such that $\left(C_{\eta} \cap E\right)^{y} \neq \varnothing$. There is a point of $\partial\left(E^{y}\right)$ between any point of $(C E)^{y}$ and any point of $E^{y}$, and therefore there exists $x \in I$ such that $x \in \partial\left(E^{y}\right)$ and $(x ; y) \in C_{\tau}$ where $0<\tau<\eta$. Consequently $\lambda_{n}\left(E \cap C_{\tau}\right)<r^{-1} \lambda_{n}\left(C_{\tau}\right)$, by (6), and so $C_{\tau} \subset A$. This would contradict (3) if $y$ belonged to $Z$, so we have shown that $\left\{y:\left(C_{\eta} \cap E\right)^{y} \neq \varnothing\right\} \subset W \cup(K \backslash Z)$. Since $\lambda_{n-1}^{*}(Z \cap K)>\left(1-\frac{1}{2} r^{-1}\right) \lambda_{n-1}(K)$, it follows that

$$
\lambda_{n-1}^{*}\left(\left\{y:\left(C_{\eta} \cap E\right)^{y}=\varnothing\right\}\right)>\left(1-r^{-1}\right) \lambda_{n-1}(K)
$$

and hence by Fubini's theorem $\lambda_{n}\left(E \cap C_{\eta}\right)<r^{-1} \lambda_{n}\left(C_{\eta}\right)$; this contradiction to (5) completes the proof.

Consider the special case where $E$ is a totally disconnected compact set in $R^{n}$ of positive $n$-measure. We adjust $E$ as above to an $x_{1}$-smooth set. In this case, the $x_{1}$-smooth adjustment is merely a reduction of $E$ by a set of measure zero since no linear interval intersects $C E$ in a set of 1 -measure zero. If $E^{*}$ is the $x_{1}$-smooth reduction of $E$, then for almost all $y \in R^{n-1}$ the set $\{x: \underline{d}(E,(x ; y))=0$ and $\bar{d}(E(x ; y))=1\}$ is residual in $\left(E^{*}\right)^{y}$ since the compactness of $\left(E^{*}\right)^{y}$ implies $\left(E^{*}\right)^{y}=\partial\left(\left(E^{*}\right)^{y}\right)$. Moreover, the set of $y$ for which $\left(E^{*}\right)^{y}$ is uncountable has positive $(n-1)$-measure. We accordingly have the following corollary to Theorem 2 .

COROLlARY 1. If $E$ is a totally disconnected compact set of positive n-measure, then $\left(\partial_{e} E\right)^{y}$ is uncountable for a set of values of $y$ of positive $(n-1)$-measure.

It is rather easy to see that the set $\partial_{e} E$ of Corollary 1 is of non- $\sigma$-finite $(n-1)$-measure. For this purpose, let $T$ be a set in $(n-1)$-space which is of finite outer Hausdorff measure, i.e. $\lambda_{0}^{n-1}(T)<\infty$. Let $N$ be a positive integer. For every $y \in T$, let $x_{1}(y)<x_{2}(y)<\cdots<x_{N}(y)$ be $N$ reals, and let

$$
\delta(y)=\min \left(x_{i}(y)-x_{i-1}(y)\right), \quad i=2, \ldots, N .
$$

For every $\delta>0$, let $T_{\delta} \subset T$ consist of those $y$ for which $\delta(y)>\delta$. For each $\eta>0$, there is a $\delta>0$ such that $\lambda_{0}^{n-1}\left(T_{\delta}\right)>\lambda_{0}^{n-1}(T)-\eta$. For each $y \in T$, let $S(y)=$ $\left\{\left(x_{1}(y), y\right), \ldots,\left(x_{N}(y), y\right)\right\}$, let $S=\bigcup_{y \in T} S(y)$ and $S_{\delta}=\bigcup_{y \in T_{\delta}} S(y)$.

For each covering of $S$ by balls of radius less than $\delta / 2$, the horizontal line at $y$ meets $N$ disjoint balls of the covering, for every $y \in S_{\delta}$. This implies that $\lambda_{0}^{n-1}(S) \geqslant$ $N\left(\lambda_{0}^{n-1}(T)-\eta\right)$. Since this holds for every $\eta>0$, we have $\lambda_{0}^{n-1}(S) \geqslant N \lambda_{0}^{n-1}(T)$.

Suppose now that $T \subset R^{n-1}$ is measurable and $\lambda^{n-1}(T)>0$. For each $y \in T$, let $S(y)$ be uncountable and let $S=\bigcup_{y \in T} S(y)$. Suppose $A \subset S$, with $\lambda_{0}^{n-1}(A)<\infty$. By the above, $A \cap S(y)$ is finite for almost all $y \in T$. Accordingly there is no sequence $\left\{A_{n}\right\}$ of subsets of $S$ such that $S=\cup_{n} A_{n}$ and $\lambda_{0}^{n-1}\left(A_{n}\right)<\infty, n=1,2, \ldots$ Thus the set $\partial_{e} E$ of Corollary 1 is of non- $\sigma$-finite $(n-1)$-measure. 
From Theorem 1 and Corollary 1 we now obtain

THEOREM 3. If $E \subset R^{n}$ is a totally disconnected compact set of positive n-measure then $\partial_{e} E$ is of non- $\sigma$-finite $(n-1)$-measure, but there are examples of such sets for which the Hausdorff dimension of $\partial_{e} E$ is $n-1$.

We benefited from very useful discussions with J. M. Marstrand at an earlier stage of this work.

\section{REFERENCES}

1. H. Federer, A note on the Gauss-Green theorem, Proc. Amer. Math. Soc. 9 (1958), 447-451.

2. A. I. Vol'pert, The spaces $B V$ and quasilinear equations, Math. USSR-Sb. 2 (1967), 225-267.

Department of Mathematics, University of California, Santa Barbara, California 93106

Department of Mathmatics, the University, Leicester, ENGland

Department of Mathematics, Purdue University, West Lafayette, Indiana 47907 